\title{
Suppression of maximal linear gluon polarization in angular asymmetries
}

\author{
Daniël Boer, ${ }^{a}$ Piet J. Mulders, ${ }^{b}$ Jian Zhou $^{c}$ and Ya-jin Zhou ${ }^{c}$ \\ ${ }^{a}$ Van Swinderen Institute for Particle Physics and Gravity, University of Groningen, \\ Nijenborgh 4, 9747 AG Groningen, The Netherlands \\ ${ }^{b}$ Nikhef and Department of Physics and Astronomy, VU University Amsterdam, \\ De Boelelaan 1081, NL-1081 HV Amsterdam, The Netherlands \\ ${ }^{c}$ School of physics \& Key Laboratory of Particle Physics and Particle Irradiation (MOE), \\ Shandong University, \\ Jinan, Shandong 250100, China \\ E-mail: d.boer@rug.nl, p.j.g.mulders@vu.nl, jzhou@sdu.edu.cn, \\ zhouyj@sdu.edu.cn
}

ABStRACT: We perform a phenomenological analysis of the $\cos 2 \phi$ azimuthal asymmetry in virtual photon plus jet production induced by the linear polarization of gluons in unpolarized $p A$ collisions. Although the linearly polarized gluon distribution becomes maximal at small $x$, TMD evolution leads to a Sudakov suppression of the asymmetry with increasing invariant mass of the $\gamma^{*}$-jet pair. Employing a small- $x$ model input distribution, the asymmetry is found to be strongly suppressed under TMD evolution, but still remains sufficiently large to be measurable in the typical kinematical region accessible at RHIC or LHC at moderate photon virtuality, whereas it is expected to be negligible in $Z / W$-jet pair production at LHC. We point out the optimal kinematics for RHIC and LHC studies, in order to expedite the first experimental studies of the linearly polarized gluon distribution through this process. We further argue that this is a particularly clean process to test the $k_{t}$-resummation formalism in the small- $x$ regime.

KEYWORDs: Perturbative QCD, Resummation

ARXIV EPRINT: 1702.08195 


\section{Contents}

1 Introduction 1

2 Theoretical setup 3

$\begin{array}{lll}3 & \text { Numerical results } & 6\end{array}$

$\begin{array}{lll}4 & \text { Summary } & 11\end{array}$

\section{Introduction}

The linearly polarized gluon distribution has received growing attention from both the small- $x$ physics and the spin physics community in recent years. It is the only polarization dependent gluon transverse momentum dependent distribution (TMD) inside an unpolarized nucleon or nucleus at leading power. The linearly polarized gluon distribution denoted as $h_{1}^{\perp g}$ was first introduced in ref. [1], and later was discussed in a model in ref. [2]. From a different point of view, it was also considered in the context of $k_{t}$ resummation [3-5]. The linearly polarized gluon distribution so far has not yet been studied experimentally. It has been proposed to probe $h_{1}^{\perp g}$ by measuring a $\cos 2 \phi$ azimuthal asymmetry for two particle production in various processes [6-9]. In all these cases, the maximal asymmetries allowed by the positivity bound constraint for the linearly polarized gluon distribution turn out to be sizable. These findings are quite promising concerning a future extraction of $h_{1}^{\perp g}$ at RHIC, LHC, or a future Electron-Ion Collider (EIC). It has also been noted that $h_{1}^{\perp g}$ affects the angular independent transverse momentum distribution of scalar or pseudoscalar particles, such as the Higgs boson [10-16] or charmonium and bottomonium states [17-21] at LHC.

Theoretical studies of the linearly polarized gluon distribution indicate that it can be quite sizable compared to the unpolarized gluon distribution. In the DGLAP formalism its small- $x$ asymptotic behavior is the same as that of the unpolarized gluon distribution, implying that it grows equally rapidly towards small $x$. In the McLerran-Venugopalan (MV) model $[22,23]$ that describes gluon saturation at small $x$, it has been found [24] that the linearly polarized gluon distribution inside a large nucleus (or nucleon) reaches its maximal value allowed by the positivity constraint for transverse momenta above the saturation scale $Q_{s}$. At low transverse momentum, the gauge link structure of the gluon TMDs becomes relevant, which is intimately connected to the process considered. For the Weizsäcker-Williams (WW) type $h_{1}^{\perp g}$ containing two (same-side) fundamental staple-like gauge links equivalent to a single adjoint staple-like gauge link, the gluon linear polarization is suppressed in the dense medium region, while for the dipole type $h_{1}^{\perp g}$ containing oppositeside fundamental staple-like gauge links equivalent to an interrupted closed Wilson loop, 
it saturates the positivity bound [24]. It is further shown in ref. [25] that the small- $x$ evolution of the dipole type polarized gluon TMD and the normal unpolarized gluon TMD is governed by the same nonlinear evolution (BK) equation. In contrast, the WW type $h_{1}^{\perp g}$ does not rise as rapidly as the WW type unpolarized gluon TMD towards small $x$ region. The phenomenological implications of the large gluon linear polarization inside a large nucleus have been explored in refs. [24-30]. The process under consideration in the present paper probes the dipole type TMDs, for which the linear gluon polarization is expected to become maximal at small $x$.

To reliably extract small- $x$ gluon TMDs in high energy scattering, it is of great importance to first establish TMD factorization. As a leading power approximation, TMD factorization is usually expected to hold at moderate to large $x$ [31-33]. At small $x$, however, higher twist contributions are equally important as the leading twist contribution because of the high gluon density. In order to arrive at an effective TMD factorization at small $x$, one first has to calculate the complete hard scattering cross section in the Color Glass Condensate (CGC) framework, which is expressed as the convolution of hard part and Wilson lines. The next step is to extrapolate the full CGC result to the correlation limit where the gluon transverse momentum is much smaller than the hard scale(s) in the process. One can then justify the use of TMD factorization at small $x$, by reducing the complete CGC result to the cross section formula derived in TMD factorization. Such an effective TMD factorization has been established in various high energy scattering processes in $e A$ and $p A$ collisions at large $N_{c}[34,35]$. This has been extended to finite $N_{c}$ and polarization dependent cases for heavy quark production in $p A$ collisions in ref. [28], as well as to other channels for two particle production in $p A$ collisions [36-38].

In this paper, we study the $\cos 2 \phi$ azimuthal asymmetry for virtual photon-jet production in the forward region in $p A$ collisions, i.e., $p+A \rightarrow \gamma^{*}+q+X$. Here, the azimuthal angle $\phi$ refers to the angle between the transverse momentum of the $\gamma^{*}-q$ pair $\left(k_{\perp}\right)$ and that of the virtual photon or the jet $\left(P_{\perp}\right)$ in the back-to-back correlation limit $\left(k_{\perp} \ll P_{\perp}\right)$. From a theoretical point of view, this is the cleanest and simplest process to access the dipole type linearly polarized gluon distribution. The back-to-back correlation limit is essential here, because only in this limit one finds a full match between the CGC result and the effective TMD factorization [24, 25]. We will present some technical details in the next section showing how to extrapolate the CGC result to the correlation limit. However, this is not yet the complete story. Due to the fact that there exists two well separated scales $k_{\perp}^{2}$ and $P_{\perp}^{2}$ in the process under consideration, improving the perturbative calculation in a systemic way requires to resum to all orders the large logarithms $\alpha_{s} \ln ^{2} P_{\perp}^{2} / k_{\perp}^{2}$ that show up in higher order corrections. It has been shown in refs. [39, 40] that such resummation can be done consistently within the CSS formalism in the saturation regime. As a result, the standard double logarithm Sudakov form factor emerges in the effective TMD factorization formula, which leads to the suppression of the asymmetry as shown below. In addition, more recent work [41] indicates that the single logarithm $\alpha_{s} \ln P_{\perp}^{2} / k_{\perp}^{2}$ also can be consistently resummed in the small- $x$ formalism at least in the dilute limit. Both the double and single logarithms are included in our phenomenological analysis of the azimuthal asymmetry following the standard CSS formalism. We note that another 
formulation of the small- $x$ Sudakov resummation exists in the literature [42], but relating the two approaches will not be attempted here.

For completeness it should be mentioned that the linearly polarized gluon distribution is not the only spin-dependent gluon distribution that is relevant at small $x$. It was found in a sequence of papers [43-46] that the Sivers gluon distribution of the dipole type is not suppressed by a full power of $x$ with respect to the unpolarized gluon TMD towards small $x$, whereas the WW type one is. Furthermore, it has been shown in refs. [47-50] that the polarization dependent five-dimensional generalized TMD inside a large nucleus could be sizable. In addition, several spin-dependent gluon TMDs inside a spin-1 target that could persist in the small- $x$ limit are identified in ref. [51]. It would be very interesting to test these theoretical expectations at RHIC, LHC or a future EIC.

The paper is structured as follows. In the next section, we describe the general theoretical framework, including justifying the use of effective TMD factorization from a CGC expression, the discussion of the associated factorization properties, and incorporating the Sudakov suppression effect. In section III, we present the numerical results for the azimuthal asymmetry in the various kinematical regions potentially accessible at RHIC and LHC. A summary of our findings and conclusions is presented in section IV.

\section{Theoretical setup}

The virtual photon-jet production in the forward region in $p A$ collision is dominated by the partonic process,

$$
q\left(x_{p} \bar{P}\right)+g\left(x P+k_{\perp}\right) \rightarrow \gamma^{*}\left(p_{1}\right)+q\left(p_{2}\right),
$$

where $x P+k_{\perp}$ is understood as the total momentum transfer through multiple gluon exchange when a quark from proton scattering off the gluon background inside a large nucleus. Typically one computes the cross section for this process using a hybrid approach [52] in which the dense target nucleus is treated as a CGC, while on the side of dilute projectile proton one uses the ordinary integrated parton distribution functions (PDFs). Although a general proof of this method is still lacking, from a practical point of view such a hybrid approach is very useful and we will employ it here. Problematic contributions causing a violation of (generalized) TMD factorization [53] are absent in this formalism. This is because one still can employ the Ward identity argument to decouple longitudinal gluon attachments from the proton side like in collinear factorization. Put differently, the factorization breaking terms are suppressed by powers of $\Lambda_{\mathrm{QCD}}^{2} / Q_{s}^{2}$ in the semi-hard region where the imbalance transverse momentum of the virtual photon-jet system is of the order of the saturation scale $Q_{s}$. For more detailed arguments why the factorization breaking effects can be avoided in the semi-hard region, we refer readers to refs. [28, 54-56].

It is straightforward to obtain the production amplitude in this hybrid approach [52],

$$
\mathcal{M}=H\left(k_{\perp}\right)\left[U\left(k_{\perp}\right)-(2 \pi)^{2} \delta^{2}\left(k_{\perp}\right)\right]
$$


with $H\left(k_{\perp}\right)$ given by,

$$
H\left(k_{\perp}\right)=\bar{u}\left(p_{2}\right)\left[(i e) \notin i \frac{x_{p} \not \vec{P}+x \not P+\not k_{\perp}}{\left(x_{p} \bar{P}+x P+k_{\perp}\right)^{2}+i \epsilon} \not p+\not p i \frac{x_{p} \not{P}-\not p_{1}}{\left(x_{p} \bar{P}-p_{1}\right)^{2}+i \epsilon}(i e) \notin\right] u\left(x_{p} \bar{P}\right),
$$

where $\varepsilon^{\mu}$ is the polarization vector of the produced virtual photon. The Wilson line $U\left(k_{\perp}\right)$ is defined as,

$$
U\left(k_{\perp}\right)=\int d^{2} x_{\perp} e^{i k_{\perp} \cdot x_{\perp}} \mathcal{P} e^{i g \int_{-\infty}^{+\infty} d x^{-} A^{+}\left(x^{-}, x_{\perp}\right)} .
$$

The above expresses that in the small- $x$ limit, the incoming quark from the proton interacts coherently with the nucleus as a whole. These interactions are summarized into Wilson lines which stretch from minus infinity to plus infinity. The next step is to extrapolate this CGC result to the correlation limit either in coordinate space $[25,34,35]$ or in momentum space $[24,28]$. Here we choose the latter and introduce the two momenta $P_{\perp}=\left(p_{1 \perp}-p_{2 \perp}\right) / 2$ and $k_{\perp}=p_{1 \perp}+p_{2 \perp}$. In the correlation limit, one has $\left|P_{\perp}\right| \simeq\left|p_{1 \perp}\right| \simeq\left|p_{2 \perp}\right| \gg\left|k_{\perp}\right|=$ $\left|p_{1 \perp}+p_{2 \perp}\right|$. The azimuthal angle between $k_{\perp}$ and $P_{\perp}$ will be denoted by $\phi$. In the correlation limit, the additional hard scale $P_{\perp}^{2}$ ensures that the hard scattering takes place locally where only a single gluon exchange from the nucleus takes part in the interaction. Multiple exchanges are power suppressed. This corresponds to a Taylor expansion of the hard part,

$$
H\left(k_{\perp}\right)=H\left(k_{\perp}=0\right)+\left.\frac{\partial H\left(k_{\perp}\right)}{\partial k_{\perp}^{i}}\right|_{k_{\perp}=0} k_{\perp}^{i}+\ldots,
$$

where the first term does not contribute and the neglected terms are suppressed by powers of $\left|k_{\perp}\right| /\left|p_{2 \perp}\right|$. The cross section is then calculated by squaring the amplitude,

$$
\left.\left.d \sigma \propto \frac{\partial H\left(k_{\perp}\right)}{\partial k_{\perp}^{i}}\right|_{k_{\perp}=0} \frac{\partial H^{*}\left(k_{\perp}\right)}{\partial k_{\perp}^{j}}\right|_{k_{\perp}=0} \int d^{2} x_{\perp} d^{2} y_{\perp} k_{\perp}^{i} k_{\perp}^{j} e^{i\left(x_{\perp}-y_{\perp}\right) \cdot k_{\perp}}\left\langle\operatorname{Tr}\left[U^{\dagger}\left(y_{\perp}\right) U\left(x_{\perp}\right)\right]\right\rangle .
$$

Using the formula,

$$
\partial^{i} U\left(x_{\perp}\right)=-i g \int_{-\infty}^{\infty} d x^{-} U\left[-\infty, x^{-}, x_{\perp}\right] \partial^{i} A^{+}\left(x^{-}, x_{\perp}\right) U\left[x^{-},+\infty, x_{\perp}\right],
$$

one can identify the soft part as the gluon TMD matrix element after partial integration. Effectively it means that in the correlation limit the incoming quark no longer interacts coherently with the nucleus throughout the process, but that the leading power effect of the interactions with the classical gluonic state are restricted to before and after the hard scattering. The net effect is an $\mathrm{SU}(3)$ color rotation of the incoming quark and the outgoing quark-virtual photon system that is encoded in the gauge links of the TMD. We parameterize the gluon TMD matrix element as,

$$
\begin{aligned}
\int d^{2} x_{\perp} d^{2} y_{\perp} e^{i\left(x_{\perp}-y_{\perp}\right) \cdot k_{\perp}}\left\langle\operatorname{Tr}\left[\partial^{i} U^{\dagger}\left(y_{\perp}\right) \partial^{i} U\left(x_{\perp}\right)\right]\right\rangle \\
\propto \frac{\delta_{\perp}^{i j}}{2} x f_{1}^{g}\left(x, k_{\perp}\right)+\left(\frac{k_{\perp}^{i} k_{\perp}^{j}}{k_{\perp}^{2}}-\frac{1}{2} \delta_{\perp}^{i j}\right) x h_{1}^{\perp g}\left(x, k_{\perp}\right),
\end{aligned}
$$


where $f_{1}^{g}\left(x, k_{\perp}\right)$ is the regular unpolarized gluon TMD. Note that the convention for $h_{1}^{\perp g}$ used here differs from [1] by a factor $k_{\perp}^{2} / 2 M_{p}^{2}$, such that positivity bound reads $\left|h_{1}^{\perp g}\left(x, k_{\perp}\right)\right| \leq f_{1}^{g}\left(x, k_{\perp}\right)$. By contracting the hard part with the tensor $\frac{\delta_{\perp}^{i j}}{2}$, one obtains the azimuthal independent cross section, while contracting the hard part with the tensor $\left(\frac{k_{\perp}^{i} k_{\perp}^{j}}{k_{\perp}^{2}}-\frac{1}{2} \delta_{\perp}^{i j}\right)$ produces a $\cos 2 \phi$ modulation. Collecting all these ingredients, we eventually arrive at the cross section formula [24],

$$
\frac{d \sigma^{p A \rightarrow \gamma^{*} q X}}{d P . S}=\sum_{q} x_{p} f_{1}^{q}\left(x_{p}\right)\left\{x f_{1}^{g}\left(x, k_{\perp}\right) H_{\mathrm{Born}}+\cos (2 \phi) x h_{1}^{\perp g}\left(x, k_{\perp}\right) H_{\mathrm{Born}}^{\cos (2 \phi)}\right\}
$$

where $f_{1}^{q}\left(x_{p}\right)$ is the quark collinear PDF of the proton and the hard coefficients are given by,

$$
\begin{aligned}
H_{\mathrm{Born}} & =\frac{\alpha_{s} \alpha_{e m} e_{q}^{2}(1-z) z^{2}}{N_{c}}\left[\frac{1+(1-z)^{2}}{\left(P_{\perp}^{2}+(1-z) Q^{2}\right)^{2}}-\frac{2 Q^{2} P_{\perp}^{2} z^{2}(1-z)}{\left(P_{\perp}^{2}+(1-z) Q^{2}\right)^{4}}\right], \\
H_{\mathrm{Born}}^{\cos (2 \phi)} & =\frac{\alpha_{s} \alpha_{e m} e_{q}^{2}(1-z) z^{2}}{N_{c}} \frac{-2 Q^{2} P_{\perp}^{2} z^{2}(1-z)}{\left(P_{\perp}^{2}+(1-z) Q^{2}\right)^{4}}
\end{aligned}
$$

which is in full agreement with that derived from TMD factorization [24].

In the above formula, the phase space factor is defined as $d P . S=d y_{q} d y_{\gamma^{*}} d^{2} P_{\perp} d^{2} k_{\perp}$, where $y_{q}$ and $y_{\gamma^{*}}$ are the rapidities of the produced quark and the virtual photon, respectively. $Q^{2}$ and $z$ are the virtual photon invariant mass and the longitudinal momentum fraction of the incoming quark carried by the virtual photon, respectively.

The next step is to resum the large logarithm $\ln P_{\perp}^{2} / k_{\perp}^{2}$ that arises from higher order corrections. An explicit one-loop calculation of the scalar particle production has shown that the $k_{t}$ resummation can be consistently done using the standard CSS formalism within the CGC effective theory framework [39]. It has been further demonstrated in ref. [40] that the double leading logarithm terms can be resummed into a Sudakov form factor for photon-jet production in the unpolarized case. More evidence that the conventional $k_{t}$ resummation procedure in general is compatible with the small- $x$ formalism has been found in ref. [41], implying that both the double leading logarithm and single leading logarithm can be resummed into the Sudakov form factor for the unpolarized case as well as the polarized case by means of the Collins-Soper evolution equation.

To facilitate resumming the large $k_{t}$ logarithm, one should Fourier transform the cross section formula to $b_{\perp}$ space and insert the Sudakov form factor following the standard CSS formalism, leading to

$$
\begin{aligned}
\frac{d \sigma^{p A \rightarrow \gamma^{*} q X}}{d P . S}= & \sum_{q} \int d^{2} b_{\perp} e^{i k_{\perp} \cdot b_{\perp}} x_{p} f_{1}^{q}\left(x_{p}, \mu_{b}^{2}\right) e^{-S\left(\mu_{b}^{2}, P_{\perp}^{2}\right)} \\
& \times\left\{x f_{1}^{g}\left(x, b_{\perp}^{2}, \mu_{b}^{2}\right) H_{\mathrm{Born}}+x h_{1}^{\perp g}\left(x, b_{\perp}^{2}, \mu_{b}^{2}\right)\left[2\left(\hat{b}_{\perp} \cdot \hat{P}_{\perp}\right)^{2}-1\right] H_{\mathrm{Born}}^{\cos (2 \phi)}\right\}
\end{aligned}
$$

where $\mu_{b}=2 e^{-\gamma_{E}} /\left|b_{\perp}\right|, \hat{b}_{\perp}=b_{\perp} /\left|b_{\perp}\right|$ and $\hat{P}_{\perp}=P_{\perp} /\left|P_{\perp}\right|$ are unit vectors. The unpolarized 
and polarized gluon TMDs in $b_{\perp}$ space are given by,

$$
\begin{aligned}
x f_{1}^{g}\left(x, b_{\perp}^{2}, \mu_{b}^{2}\right) & =\int \frac{d^{2} k_{\perp}}{(2 \pi)^{2}} e^{i k_{\perp} \cdot b_{\perp}} x f_{1}^{g}\left(x, k_{\perp}, \mu_{b}^{2}\right), \\
x h_{1}^{\perp g}\left(x, b_{\perp}^{2}, \mu_{b}^{2}\right) & =-\int \frac{d\left|k_{\perp}\right|}{2 \pi} J_{2}\left(\left|b_{\perp}\right|\left|k_{\perp}\right|\right) x h_{1}^{\perp g}\left(x, k_{\perp}, \mu_{b}^{2}\right),
\end{aligned}
$$

where the standard $\zeta$ parameter is chosen identical to the renormalization scale $\mu_{b}$ and not shown here. At tree level the Sudakov factor is zero, in which case one recovers eq. (2.9) after Fourier transforming back to $k_{\perp}$ space. At one-loop order, the perturbative Sudakov form factor (valid for sufficiently small $b_{\perp}$ ) takes the form,

$$
S\left(\mu_{b}^{2}, P_{\perp}^{2}\right)=\int_{\mu_{b}}^{\left|P_{\perp}\right|} \frac{d \mu}{\mu} \alpha_{s}(\mu)\left(\frac{C_{F}+C_{A}}{\pi} \ln \frac{P_{\perp}^{2}}{\mu^{2}}-\frac{C_{F}}{\pi} \frac{3}{2}-\frac{C_{A}}{\pi} \frac{11-2 n_{f} / C_{A}}{6}\right)
$$

where the $C_{F}$ part receives a contribution from a gluon radiated off the quark line, while the $C_{A}$ part is generated from the Collins-Soper type small- $x$ gluon TMD evolution. We will discuss the nonperturbative Sudakov factor in the next section.

To avoid having to deal with a three scale problem, we restrict to the kinematical region where $Q^{2}$ is of the order of $P_{\perp}^{2}$. This happens to be the optimal region to probe $h_{1}^{\perp g}$ as suggested by our numerical estimation.

\section{$3 \quad$ Numerical results}

To evolve the gluon TMDs to a higher scale, one has to first determine the gluon TMDs at an initial scale. It is common to compute the gluon distributions in the MV model and use them as the initial condition for small $x$ evolution. At RHIC energy, the typical longitudinal momentum fraction carried by gluon probed in the process under consideration is around $x \sim 0.01$, which we consider to be sufficiently small to apply a small- $x$ input distribution. Because of the limited $x$ range probed at RHIC, we do not include small- $x$ evolution. At LHC this may become relevant though.

We will use the MV model results as the initial input for the Collins-Soper type evolution. In the MV model, the dipole type unpolarized and linearly polarized gluon TMDs are identical [24],

$$
x h_{1}^{\perp g}\left(x, k_{\perp}\right)=x f_{1}^{g}\left(x, k_{\perp}\right)=\frac{k_{\perp}^{2} N_{c}}{2 \pi^{2} \alpha_{s}} S_{\perp} \int \frac{d^{2} b_{\perp}}{(2 \pi)^{2}} e^{-i k_{\perp} \cdot b_{\perp}} e^{-\frac{b_{\perp}^{2} Q_{s}^{2}}{4}},
$$

where $S_{\perp}$ denotes the transverse area of a large nucleus. To facilitate numerical estimation, we reexpress it as [57],

$$
x f_{1}^{g}\left(x, k_{\perp}\right)=\frac{k_{\perp}^{2} A x f_{1, p}^{g}(x)}{Q_{s}^{2}} \int \frac{d^{2} b_{\perp}}{(2 \pi)^{2}} e^{-i k_{\perp} \cdot b_{\perp}} e^{-\frac{b_{\perp}^{2} Q_{s}^{2}}{4}}
$$


with $f_{1, p}^{g}(x)$ being the standard gluon PDF in a nucleon, for which we will employ the MSTW 2008 LO PDF set. Substituting eq. (3.2) to eq. (2.11) and eq. (2.12), one obtains,

$$
\begin{aligned}
x f_{1}^{g}\left(x, b_{\perp}^{2}, \mu^{2} \equiv Q_{s}^{2}\right) & =A x f_{1, p}^{g}\left(x, \mu^{2} \equiv Q_{s}^{2}\right) \frac{1}{(2 \pi)^{2}}\left(1-\frac{Q_{s}^{2} b_{\perp}^{2}}{4}\right) e^{-\frac{Q_{s}^{2} b_{\perp}^{2}}{4}}, \\
x h_{1}^{\perp g}\left(x, b_{\perp}^{2}, \mu^{2} \equiv Q_{s}^{2}\right) & =-A x f_{1, p}^{g}\left(x, \mu^{2} \equiv Q_{s}^{2}\right) \frac{1}{(2 \pi)^{2}} \frac{Q_{s}^{2} b_{\perp}^{2}}{4} e^{-\frac{Q_{s}^{2} b_{\perp}^{2}}{4}} .
\end{aligned}
$$

In arriving at the above formulas, we have neglected the dependence of $Q_{s}^{2}$ on $b_{\perp}^{2}$ that is usually considered as a good approximation at low $k_{\perp}$, although it leads to an incorrect perturbative tail at high transverse momentum [35]. In the current case, the correct power law tail automatically develops after taking into account TMD evolution. It should be also pointed out that the renormalization scale and the parameter $\zeta$ have been chosen to be $Q_{s}^{2}$, at which scale the MV model expressions are assumed to hold.

Before computing the asymmetry, it would be interesting to first investigate how the linearly gluon polarization is affected by TMD evolution. By solving the Collins-Soper equation, the unpolarized and polarized gluon TMDs at the scales $P_{\perp}^{2}$ read,

$$
\begin{aligned}
x f_{1}^{g}\left(x, k_{\perp}, \mu^{2}=P_{\perp}^{2}\right) & =\int d\left|b_{\perp}\right|\left|b_{\perp}\right| 2 \pi J_{0}\left(\left|k_{\perp}\right|\left|b_{\perp}\right|\right) e^{-S_{A}\left(\mu_{b}^{2}, P_{\perp}^{2}\right)} x f_{1}^{g}\left(x, b_{\perp}^{2}, \mu_{b}^{2}\right), \\
x h_{1}^{\perp g}\left(x, k_{\perp}, \mu^{2}=P_{\perp}^{2}\right) & =-\int d\left|b_{\perp}\right|\left|b_{\perp}\right| 2 \pi J_{2}\left(\left|k_{\perp}\right|\left|b_{\perp}\right|\right) e^{-S_{A}\left(\mu_{b}^{2}, P_{\perp}^{2}\right)} x h_{1}^{\perp g}\left(x, b_{\perp}^{2}, \mu_{b}^{2}\right),
\end{aligned}
$$

and similar expressions holds at the scale $Q_{s}^{2}$ after replacing $P_{\perp}^{2}$ by $Q_{s}^{2}$. For the expressions at $Q_{s}^{2}$ we will then use the MV model expressions. The gluonic part of the perturbative Sudakov factor $S_{A}\left(\mu_{b}^{2}, P_{\perp}^{2}\right)$ at one-loop order takes the form,

$$
S_{A}\left(\mu_{b}^{2}, P_{\perp}^{2}\right)=\frac{C_{A}}{2 \pi} \int_{\mu_{b}^{2}}^{P_{\perp}^{2}} \frac{d \mu^{2}}{\mu^{2}} \alpha_{s}(\mu)\left[\ln \frac{P_{\perp}^{2}}{\mu^{2}}-\frac{11-2 n_{f} / C_{A}}{6}\right],
$$

Using the above relations, it follows that

$$
\begin{aligned}
& x f_{1}^{g}\left(x, k_{\perp}, \mu^{2}=P_{\perp}^{2}\right)=\int d\left|b_{\perp}\right|\left|b_{\perp}\right| 2 \pi J_{0}\left(\left|k_{\perp}\right|\left|b_{\perp}\right|\right) \\
& x h^{-S_{A}\left(\mu_{b}^{2}, P_{\perp}^{2}\right)+S_{A}\left(\mu_{b}^{2}, Q_{s}^{2}\right)} x f_{1}^{g}\left(x, b_{\perp}^{2}, \mu^{2}=Q_{s}^{2}\right), \\
& x\left(x, k_{\perp}, \mu^{2}=P_{\perp}^{2}\right)=-\int d\left|b_{\perp}\right|\left|b_{\perp}\right| 2 \pi J_{2}\left(\left|k_{\perp}\right|\left|b_{\perp}\right|\right) \\
& \times e^{-S_{A}\left(\mu_{b}^{2}, P_{\perp}^{2}\right)+S_{A}\left(\mu_{b}^{2}, Q_{s}^{2}\right)} x h_{1}^{\perp g}\left(x, b_{\perp}^{2}, \mu^{2}=Q_{s}^{2}\right),
\end{aligned}
$$

where the Sudakov factor $-S_{A}\left(\mu_{b}^{2}, P_{\perp}^{2}\right)+S_{A}\left(\mu_{b}^{2}, Q_{s}^{2}\right)$ is the same as that used for TMD evolution from a fixed scale [58]. Note that this is not simply $S_{A}\left(P_{\perp}^{2}, Q_{s}^{2}\right)$, due to the double log nature of the expressions.

The above expressions for the Sudakov factor are valid in the perturbative region of small $b_{\perp}$. Since we are mostly interested in the semi-hard region $\left|k_{\perp}\right| \sim Q_{s}$ where the contributions from large $b_{\perp}$ could be important when performing Fourier transform, 
following the standard treatment, we introduce a non-perturbative Sudakov factor, for both $\mu^{2}=P_{\perp}^{2}$ and $\mu^{2}=Q_{s}^{2}$ :

$$
-S_{A}\left(\mu_{b}^{2}, \mu^{2}\right) \rightarrow-S_{A}\left(\mu_{b *}^{2}, \mu^{2}\right)-S^{N P}\left(b_{\perp}^{2}, \mu^{2}\right),
$$

where $\mu_{b *}^{2}$ is defined as $\mu_{b *}^{2}=4 e^{-2 \gamma_{E}} / b_{\perp *}^{2}$, with $b_{\perp *}$ given by

$$
b_{\perp *}=\frac{b_{\perp}}{\sqrt{1+b_{\perp}^{2} / b_{\max }^{2}}} \leq b_{\max },
$$

and the parametrization for the non-perturbative Sudakov factor will be taken (based on [59]) as

$$
S_{A, q}^{N P}\left(b_{\perp}^{2}, \mu^{2}\right)=S_{A, g}^{N P}\left(b_{\perp}^{2}, \mu^{2}\right) \frac{C_{F}}{C_{A}}=\frac{1}{2}\left(g_{1}+g_{2} \ln \frac{\mu}{2 Q_{0}}+2 g_{1} g_{3} \ln \frac{10 x x_{0}}{x_{0}+x}\right) b_{\perp}^{2},
$$

with $b_{\max }=1.5 \mathrm{GeV}^{-1}, g_{1}=0.201 \mathrm{GeV}^{2}, g_{2}=0.184 \mathrm{GeV}^{2}, g_{3}=-0.129, x_{0}=$ $0.009, Q_{0}=1.6 \mathrm{GeV}$. To smoothly match to the large transverse momentum region, we also regulate the very small $b_{\perp}$ behavior of the Sudakov factor by the replacement of $\mu_{b}$ by $[15,60,61]$

$$
\mu_{b}^{\prime}\left(\mu^{2}\right)=\frac{1}{\sqrt{b_{\perp}^{2} /\left(4 e^{-2 \gamma_{E}}\right)+1 / \mu^{2}}} .
$$

In our numerical estimation, we used the one-loop running coupling constant $\alpha_{s}$, with $n_{f}=3$ and $\Lambda_{\mathrm{QCD}}=216 \mathrm{MeV}$. The saturation scale is further fixed using the GBW model [62],

$$
Q_{s}^{2}(x)=(1 \mathrm{GeV})^{2} A^{1 / 3}\left(\frac{x_{0}}{x}\right)^{0.3} \quad \text { with } \quad x_{0}=3 \times 10^{-4},
$$

where the atomic number $A$ is chosen to be $A=197$ for RHIC, but $A=208$ for LHC hardly makes a difference. It results in $Q_{s}^{2} \sim 2 \mathrm{GeV}^{2}$ for RHIC in the kinematical regions under consideration.

With these ingredients, we are ready to perform the numerical study of the evolved gluon TMDs including both unpolarized and linearly polarized gluons. We evolve the gluon TMDs from the saturation scale where the MV results are used as the initial input up to the scales $\mu^{2}=(6 \mathrm{GeV})^{2}$ and $\mu^{2}=(15 \mathrm{GeV})^{2}$. As can be seen from figure 1 , the shape of the unpolarized gluon TMD at low $k_{\perp}$ is significantly changed by evolution. One further observes that the linearly polarized gluon distribution evolves very fast and is suppressed with increasing energy. Since the azimuthal asymmetry induced by the linearly polarized gluon distribution is proportional to the ratio $R=h_{1}^{\perp g} / f_{1}^{g}$, it is instructive to plot this ratio as function of $k_{\perp}$ at different scales in figure 2 . The dotted blue line represents the ratio computed from the MV model, which is identical to 1 at any value of $k_{\perp}$. That simple relation between the unpolarized gluon TMD and the linearly polarized gluon TMD still holds after taking into account small $x$ evolution [25], whereas it significantly deviates from it after energy evolution as shown in figure 2 . The ratio initially grows with increasing $k_{\perp}$ until it reaches a maximal value at a transverse momentum of about two times the saturation scale and then decreases at high transverse momentum. In the typical 


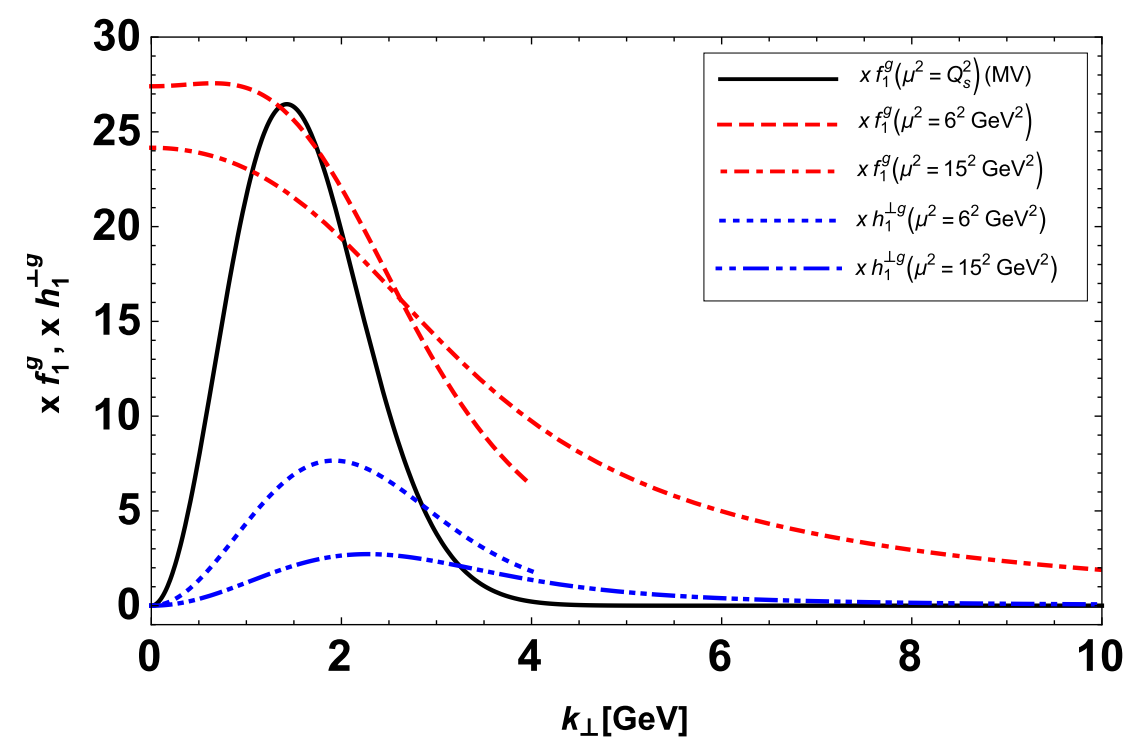

Figure 1. The unpolarized and linearly polarized gluon TMDs as function of $k_{\perp}$ at different scales, at $x=0.01$, using the MV model as input.

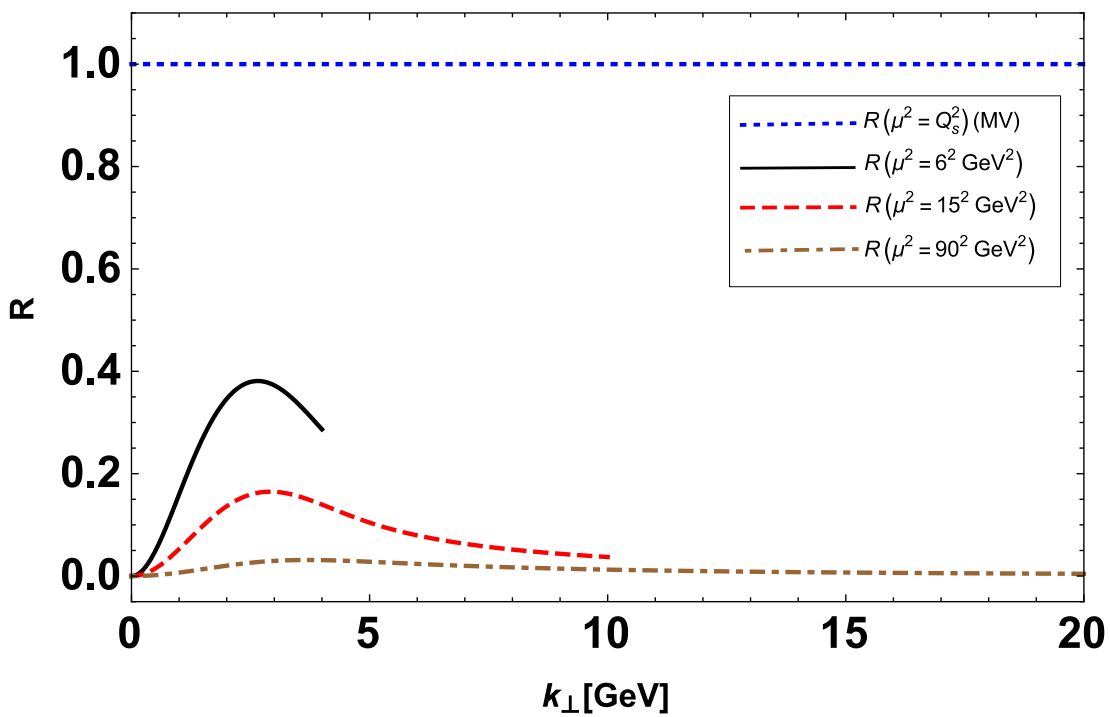

Figure 2. The ratio $R=h_{1}^{\perp g} / f_{1}^{g}$ as function of $k_{\perp}$, at $x=0.01$ for $\mu=6,15$ and $90 \mathrm{GeV}$.

kinematical region accessible at RHIC, the maximal value of the ratio is slightly less than 0.4. We also plot a curve for the ratio at the scale $\mu^{2}=(90 \mathrm{GeV})^{2}$ because it is relevant for studying $\cos 2 \phi$ asymmetry for $Z$-jet pair production in $p p$ or $p A$ collisions at LHC. Judging from this curve for $R$, we conclude that it is rather challenging to measure the mentioned azimuthal asymmetry at LHC in this process (and likely in $W$-jet pair production as well). Needless to say, $\gamma^{*}$-jet pair production at lower $\gamma^{*}$ virtuality should be feasible at LHC. There the $x$ values typically are much smaller, but $Q_{s}^{2}$ is only about a factor of 2 larger than at RHIC, requiring $P_{\perp}$ to be larger than say $10 \mathrm{GeV}$, allowing it to still be selected 

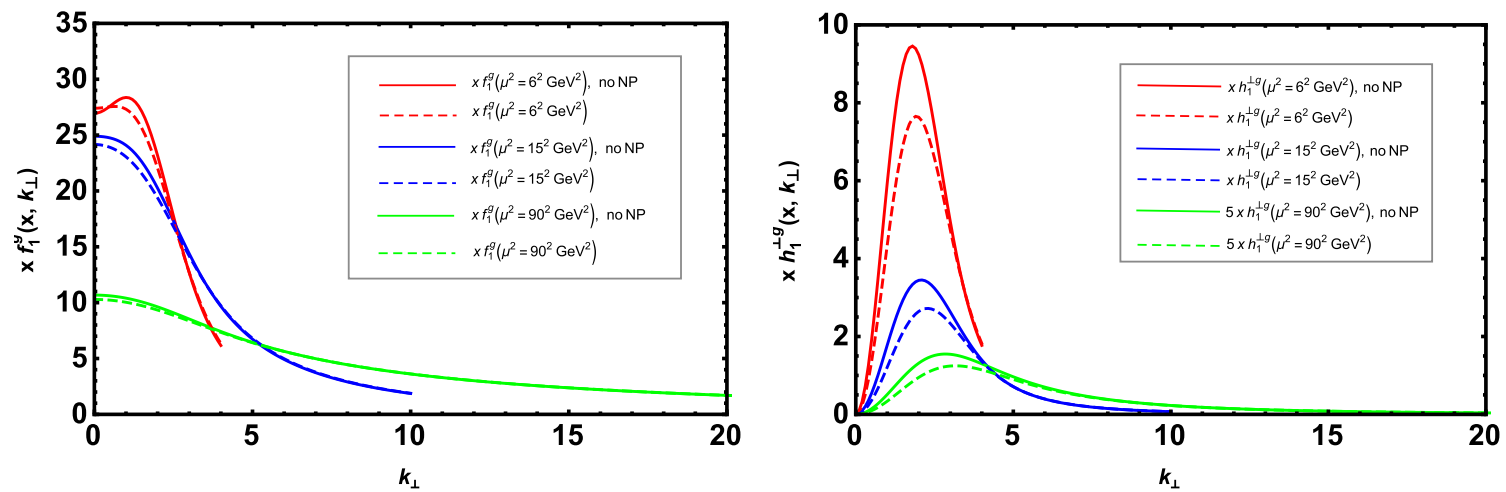

Figure 3. The unpolarized and the linearly polarized gluon TMDs with different Sudakov factor at different scales.

far below $M_{Z}$. We will also show some results for LHC below.

The above results depend on the adopted treatment of the non-perturbative $b$-region and this dependence will be more pronounced at lower energies. Rather than adopting a different treatment to assess the uncertainty, we compare to the results without any non-perturbative treatment. The results are shown in figure 3 , which shows the evolved gluon TMDs evaluated with the $b_{*}$-prescription and a nonperturbative Sudakov factor included, compared to the ones evaluated with only a purely perturbative ${ }^{1}$ Sudakov factor $e^{-\left[S_{A}\left(\mu_{b}^{2}, \mu^{2}\right)-S_{A}\left(\mu_{b}^{2}, Q_{s}^{2}\right)\right]}$. As can be seen from figure 3 , the deviation decreases with increasing energy scale. At $\mu^{2}=90^{2} \mathrm{GeV}^{2}$, the dependence of the gluon TMDs on the non-perturbative input is almost completely washed out. The deviations at low transverse momentum for lower energy scales is larger ( $25 \%$ correction) as expected, because larger $b$ values contribute, but this deviation will partly cancel in the asymmetry ratio. We conclude that it will not alter our model results for the asymmetry much and hence our conclusions, despite the inevitable uncertainty from non-perturbative contributions at lower energies.

The numerical results for the computed azimuthal asymmetry in the different kinematical regions are presented in figures 4-6. Here, the azimuthal asymmetry, i.e., the average value of $\cos (2 \phi)$, is defined as,

$$
\langle\cos (2 \phi)\rangle=\frac{\int \frac{d \sigma}{d P . S} d \phi \cos (2 \phi)}{\int \frac{d \sigma}{d P . S} d \phi}
$$

As explained in the above, to avoid dealing with the three scales problem, we choose $Q^{2}$ to be of the order of $P_{\perp}^{2}$ which sets the hard scale $\mu^{2}=P_{\perp}^{2}$ when performing energy evolution. We found that the most optimistic rapidity ranges for measuring this azimuthal asymmetry at RHIC energy $(\sqrt{s}=200 \mathrm{GeV})$ are $y_{\gamma^{*}}[1.5,2], y_{q}[0.5,1.5]$. In these rapidity ranges, figure 4 shows the asymmetry as function of $k_{\perp}$ for two different $Q^{2}$ ranges at $P_{\perp}=6 \mathrm{GeV}$. The asymmetry reach a maximal value of $6 \%$ for $Q^{2}[55,80]$ around $k_{\perp}=3 \mathrm{GeV}$. Note that the

\footnotetext{
${ }^{1}$ The perturbative curves labelled "no NP" do include the mentioned very small $b$ regulator in order to ensure the correct perturbative result for the integrated (collinear) case, cf. e.g. [63, 64]. It also ensures suppression of the NP contribution at large $k_{\perp}$.
} 


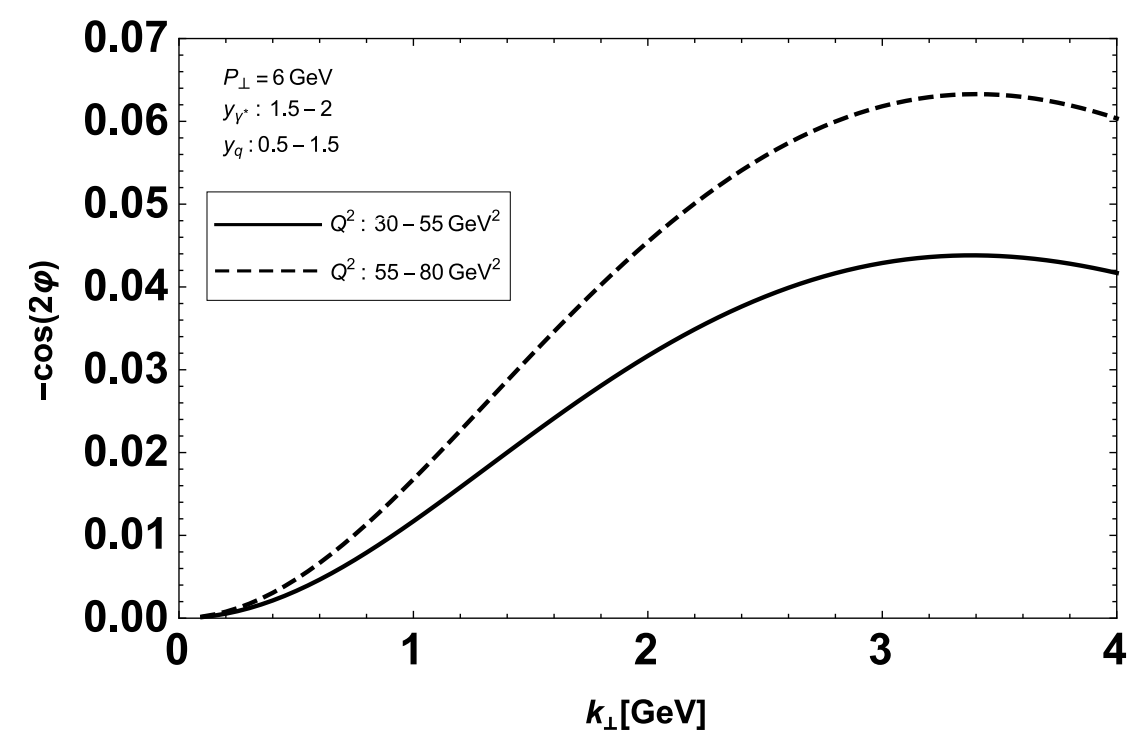

Figure 4. Estimates of the azimuthal asymmetry as function of $k_{\perp}$, calculated at $P_{\perp}=6 \mathrm{GeV}$, for the different $Q^{2}$ ranges at the center mass energy $\sqrt{s}=200 \mathrm{GeV}$. The quark and virtual photon rapidities are integrated over the regions $y_{q}[1.5,2]$ and $y_{\gamma^{*}}[0.5,1.5]$.

corresponding longitudinal momentum fraction of the gluon $x$ is in the region $[0.008,0.03]$ where the MV model results are only borderline justified at best. At LHC the situation is better in this respect, but here we just aim to illustrate the effect of TMD evolution on an observable that in principle probes the linear polarization distribution that becomes maximal at small $x$. Sudakov suppression shows that the observable asymmetry is far from maximal.

For the same rapidity regions, we also plot the asymmetry as function of $P_{\perp}$ with $Q^{2}$ chosen to be $Q^{2}=P_{\perp}^{2}$ and $Q^{2}=\frac{1}{2} P_{\perp}^{2}$, with $k_{\perp}=2.5 \mathrm{GeV}$. One sees that there is a relatively mild dependence of the asymmetry on $P_{\perp}$. Figure 5 shows that the asymmetry grows with increasing virtual photon rapidity. However, a virtual photon rapidity larger than 2 is not reachable at RHIC energy for the kinematical region under consideration. Finally, the asymmetry at LHC energy plotted in figure 6 is similar but about a factor 2-3 smaller than that at RHIC.

\section{Summary}

At small $x$ the linearly polarized gluon TMD is expected to be comparable in size to the unpolarized gluon TMD, reflecting that the Color Glass Condensate can be highly polarized. In the MV model, the linearly polarized gluon TMD saturates the positivity bound for the dipole case, which means that it is in fact identical to the unpolarized gluon TMD. This relation persists under small- $x$ evolution. Such a large effect is very promising for its experimental investigation, especially since the linearly polarized gluon TMD has not been studied experimentally thus far. From a theoretical point of view, the cleanest channel 


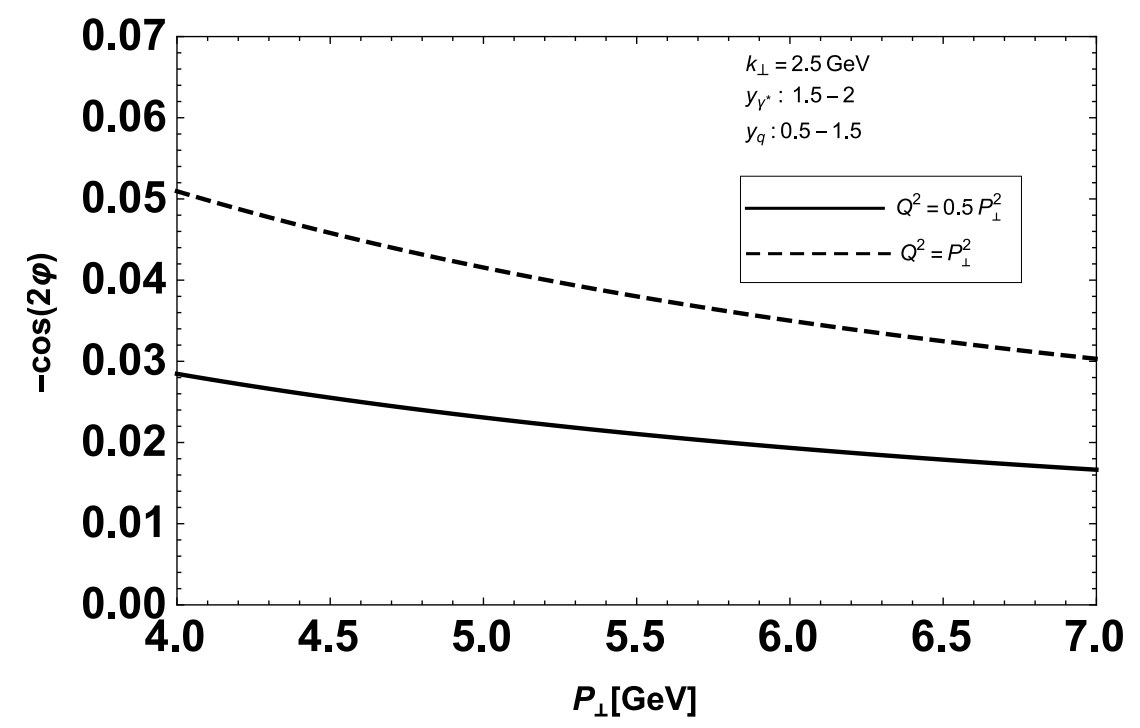

Figure 5. Estimates of the azimuthal asymmetry as function of $P_{\perp}$ with $Q^{2}=P_{\perp}^{2}$ and $Q^{2}=0.5 P_{\perp}^{2}$, at $k_{\perp}=2.5 \mathrm{GeV}$ and $\sqrt{s}=200 \mathrm{GeV}$. The quark and virtual photon rapidities are integrated over the regions $y_{q}[1.5,2]$ and $y_{\gamma^{*}}[0.5,1.5]$.

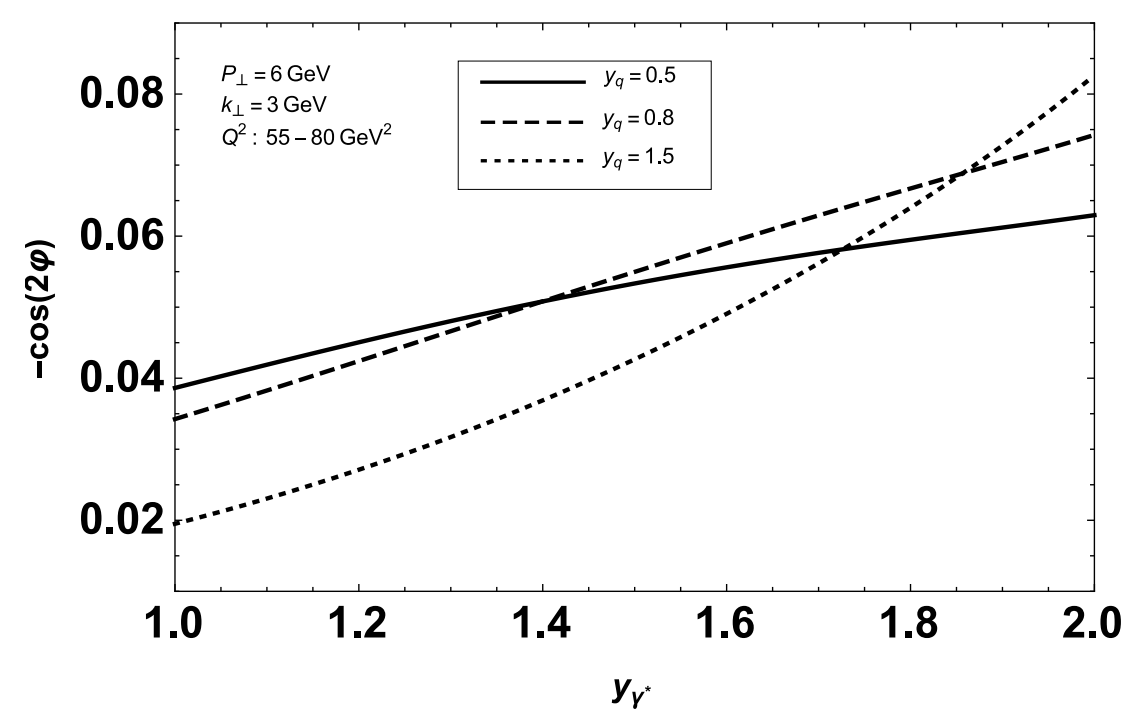

Figure 6. The azimuthal asymmetry evaluated at $k_{\perp}=3 \mathrm{GeV}, P_{\perp}=6 \mathrm{GeV}, \sqrt{s}=200 \mathrm{GeV}$, and for the different quark rapidities $y_{q}=0.5,0.8,1.5$, respectively. $Q^{2}$ is integrated over the region $[55,80] \mathrm{GeV}^{2}$. 


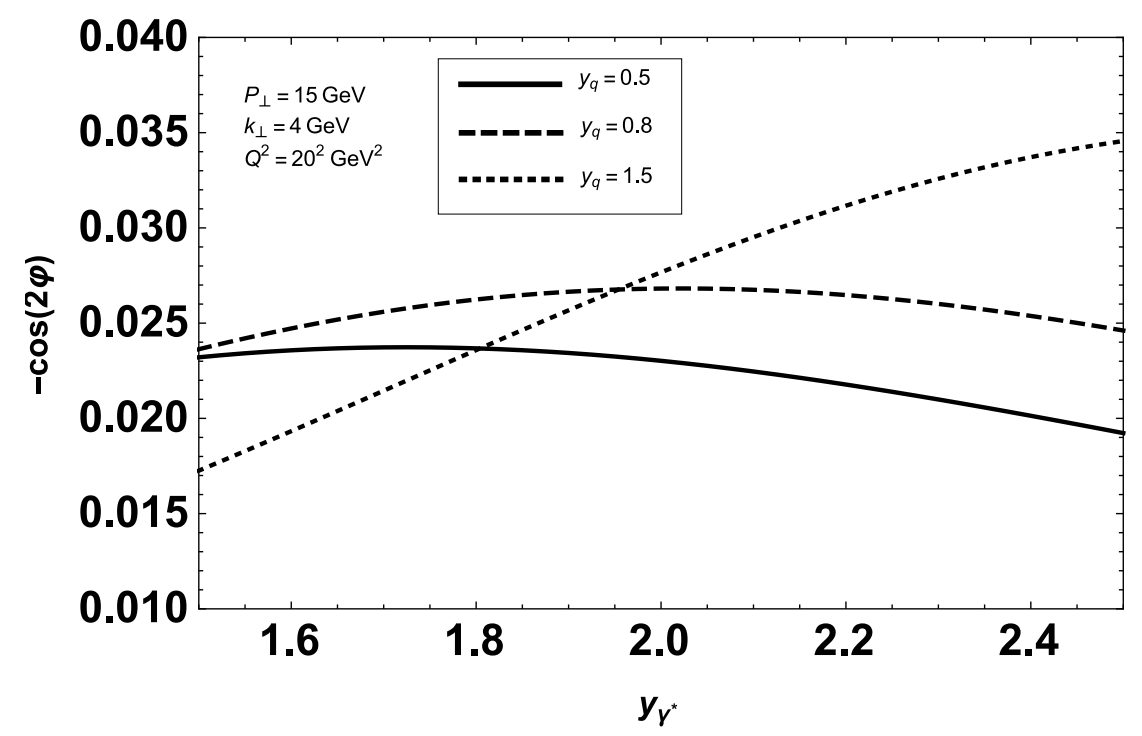

Figure 7. The azimuthal asymmetry evaluated at $k_{\perp}=4 \mathrm{GeV}, P_{\perp}=15 \mathrm{GeV}, Q=20 \mathrm{GeV}$, $\sqrt{s}=5.02 \mathrm{TeV}$ and for the different quark rapidities $y_{q}=0.5,0.8,1.5$, respectively.

to probe the dipole type linearly polarized gluon TMD is the $\cos 2 \phi$ azimuthal asymmetry in virtual photon-jet pair production in $p A$ collisions, which can be studied at RHIC and LHC.

Despite that there is no reason to expect the asymmetry to be small at small $x$, given the maximal size of the linearly polarized gluon TMD, we find that the effect of the linear gluon polarization is strongly suppressed due to TMD evolution effects. In this paper, we have presented numerical estimations of the ratio between the linearly polarized gluon TMD and the unpolarized gluon TMD and of the azimuthal asymmetry taking into account TMD evolution. The ratio after evolution peaks at a transverse momentum on the order of the saturation scale, where its maximal value for instance at scale $\mu^{2}=36 \mathrm{GeV}^{2}$ is about 0.4 and at $\mu^{2}=M_{Z}^{2}$ only on the few percent level. Despite that the linear gluon TMD enters just once in the $\cos 2 \phi$ asymmetry, the Sudakov suppression of the asymmetry is much stronger than the ratio of TMDs would suggest. For the typical kinematic regions accessible at RHIC, the maximal size of the azimuthal asymmetry is found to be around $7 \%$. For such values experimental measurements at RHIC would still seem feasible though. We note that these values do depend on the input distributions we have chosen, but nevertheless we expect these results to give a realistic reflection of the amount of Sudakov suppression for this observable. The situation for LHC is slightly worse, with 2-3\% asymmetries, but employing the small- $x$ model as a starting point is more justified in this case. We have also found that the azimuthal asymmetry in jet- $Z / W$ production at LHC is almost completely washed out by the Collins-Soper evolution effect.

In conclusion, the experimental study of this asymmetry at RHIC seems the most promising option and, despite the strong Sudakov suppression, may allow to test the $k_{t^{-}}$ resummation formalism in the small- $x$ regime and the theoretical expectation that the Color Glass Condensate state is in fact polarized. 


\section{Acknowledgments}

J. Zhou thanks Andreas Metz for suggesting to study the observable numerically. J. Zhou has been supported by the National Science Foundations of China under Grant No. 11675093, and by the Thousand Talents Plan for Young Professionals. Ya-jin Zhou has been supported by the National Science Foundations of China under Grant No. 11375104 and No. 11675092. This research has been partially supported by the EU "Ideas" program QWORK (contract 320389).

Open Access. This article is distributed under the terms of the Creative Commons Attribution License (CC-BY 4.0), which permits any use, distribution and reproduction in any medium, provided the original author(s) and source are credited.

\section{References}

[1] P.J. Mulders and J. Rodrigues, Transverse momentum dependence in gluon distribution and fragmentation functions, Phys. Rev. D 63 (2001) 094021 [hep-ph/0009343] [INSPIRE].

[2] S. Meissner, A. Metz and K. Goeke, Relations between generalized and transverse momentum dependent parton distributions, Phys. Rev. D 76 (2007) 034002 [hep-ph/0703176] [INSPIRE].

[3] P.M. Nadolsky, C. Balázs, E.L. Berger and C.P. Yuan, Gluon-gluon contributions to the production of continuum diphoton pairs at hadron colliders, Phys. Rev. D 76 (2007) 013008 [hep-ph/0702003] [INSPIRE].

[4] S. Mantry and F. Petriello, Factorization and resummation of Higgs boson differential distributions in soft-collinear effective theory, Phys. Rev. D 81 (2010) 093007 [arXiv:0911.4135] [INSPIRE].

[5] S. Catani and M. Grazzini, QCD transverse-momentum resummation in gluon fusion processes, Nucl. Phys. B 845 (2011) 297 [arXiv:1011.3918] [INSPIRE].

[6] D. Boer, P.J. Mulders and C. Pisano, Dijet imbalance in hadronic collisions, Phys. Rev. D 80 (2009) 094017 [arXiv: 0909.4652] [INSPIRE].

[7] D. Boer, S.J. Brodsky, P.J. Mulders and C. Pisano, Direct probes of linearly polarized gluons inside unpolarized hadrons, Phys. Rev. Lett. 106 (2011) 132001 [arXiv:1011.4225] [INSPIRE].

[8] J.-W. Qiu, M. Schlegel and W. Vogelsang, Probing gluonic spin-orbit correlations in photon pair production, Phys. Rev. Lett. 107 (2011) 062001 [arXiv:1103.3861] [INSPIRE].

[9] C. Pisano, D. Boer, S.J. Brodsky, M.G.A. Buffing and P.J. Mulders, Linear polarization of gluons and photons in unpolarized collider experiments, JHEP 10 (2013) 024 [arXiv: 1307.3417] [INSPIRE].

[10] P. Sun, B.-W. Xiao and F. Yuan, Gluon distribution functions and Higgs boson production at moderate transverse momentum, Phys. Rev. D 84 (2011) 094005 [arXiv:1109.1354] [INSPIRE].

[11] D. Boer, W.J. den Dunnen, C. Pisano, M. Schlegel and W. Vogelsang, Linearly polarized gluons and the Higgs transverse momentum distribution, Phys. Rev. Lett. 108 (2012) 032002 [arXiv:1109.1444] [INSPIRE]. 
[12] J. Wang, C.S. Li, Z. Li, C.P. Yuan and H.T. Li, Improved resummation prediction on Higgs production at hadron colliders, Phys. Rev. D 86 (2012) 094026 [arXiv:1205.4311] [INSPIRE].

[13] D. Boer, W.J. den Dunnen, C. Pisano and M. Schlegel, Determining the Higgs spin and parity in the diphoton decay channel, Phys. Rev. Lett. 111 (2013) 032002 [arXiv:1304.2654] [INSPIRE].

[14] S. Catani, L. Cieri, D. de Florian, G. Ferrera and M. Grazzini, Universality of transverse-momentum resummation and hard factors at the NNLO, Nucl. Phys. B 881 (2014) 414 [arXiv:1311.1654] [INSPIRE].

[15] D. Boer and W.J. den Dunnen, TMD evolution and the Higgs transverse momentum distribution, Nucl. Phys. B 886 (2014) 421 [arXiv:1404.6753] [INSPIRE].

[16] M.G. Echevarria, T. Kasemets, P.J. Mulders and C. Pisano, QCD evolution of (un)polarized gluon TMDPDFs and the Higgs $q_{T}$-distribution, JHEP 07 (2015) 158 [Erratum ibid. 05 (2017) 073] [arXiv: 1502.05354] [INSPIRE].

[17] D. Boer and C. Pisano, Polarized gluon studies with charmonium and bottomonium at LHCb and AFTER, Phys. Rev. D 86 (2012) 094007 [arXiv: 1208.3642] [INSPIRE].

[18] J.P. Ma, J.X. Wang and S. Zhao, Transverse momentum dependent factorization for quarkonium production at low transverse momentum, Phys. Rev. D 88 (2013) 014027 [arXiv:1211.7144] [INSPIRE].

[19] W.J. den Dunnen, J.P. Lansberg, C. Pisano and M. Schlegel, Accessing the transverse dynamics and polarization of gluons inside the proton at the $\mathrm{LHC}$,

Phys. Rev. Lett. 112 (2014) 212001 [arXiv:1401.7611] [INSPIRE].

[20] G.-P. Zhang, Probing transverse momentum dependent gluon distribution functions from hadronic quarkonium pair production, Phys. Rev. D 90 (2014) 094011 [arXiv:1406.5476] [INSPIRE].

[21] J.P. Ma and C. Wang, QCD factorization for quarkonium production in hadron collisions at low transverse momentum, Phys. Rev. D 93 (2016) 014025 [arXiv:1509.04421] [InSPIRE].

[22] L.D. McLerran and R. Venugopalan, Computing quark and gluon distribution functions for very large nuclei, Phys. Rev. D 49 (1994) 2233 [hep-ph/9309289] [INSPIRE].

[23] L.D. McLerran and R. Venugopalan, Gluon distribution functions for very large nuclei at small transverse momentum, Phys. Rev. D 49 (1994) 3352 [hep-ph/9311205] [INSPIRE].

[24] A. Metz and J. Zhou, Distribution of linearly polarized gluons inside a large nucleus, Phys. Rev. D 84 (2011) 051503 [arXiv:1105.1991] [INSPIRE].

[25] F. Dominguez, J.-W. Qiu, B.-W. Xiao and F. Yuan, On the linearly polarized gluon distributions in the color dipole model, Phys. Rev. D 85 (2012) 045003 [arXiv:1109.6293] [INSPIRE].

[26] A. Schäfer and J. Zhou, Higgs boson production in high energy proton-nucleus collisions, Phys. Rev. D 85 (2012) 114004 [arXiv:1203.1534] [INSPIRE].

[27] T. Liou, Color-neutral heavy particle production in nucleus-nucleus collisions in the quasi-classical approximation, Nucl. Phys. A 897 (2013) 122 [arXiv:1206.6123] [INSPIRE].

[28] E. Akcakaya, A. Schäfer and J. Zhou, Azimuthal asymmetries for quark pair production in pA collisions, Phys. Rev. D 87 (2013) 054010 [arXiv: 1208.4965] [INSPIRE]. 
[29] A. Dumitru, T. Lappi and V. Skokov, Distribution of linearly polarized gluons and elliptic azimuthal anisotropy in deep inelastic scattering dijet production at high energy, Phys. Rev. Lett. 115 (2015) 252301 [arXiv: 1508.04438] [INSPIRE].

[30] A. Dumitru and V. Skokov, $\cos (4 \varphi)$ azimuthal anisotropy in small-x DIS dijet production beyond the leading power TMD limit, Phys. Rev. D 94 (2016) 014030 [arXiv:1605.02739] [INSPIRE].

[31] J.C. Collins and D.E. Soper, Back-to-back jets in QCD, Nucl. Phys. B 193 (1981) 381 [Erratum ibid. B 213 (1983) 545] [INSPIRE].

[32] J.C. Collins and D.E. Soper, Parton distribution and decay functions, Nucl. Phys. B 194 (1982) 445 [inSPIRE].

[33] J.C. Collins, D.E. Soper and G.F. Sterman, Transverse momentum distribution in Drell-Yan pair and $W$ and $Z$ boson production, Nucl. Phys. B 250 (1985) 199 [INSPIRE].

[34] F. Dominguez, B.-W. Xiao and F. Yuan, $k_{t}$-factorization for hard processes in nuclei, Phys. Rev. Lett. 106 (2011) 022301 [arXiv:1009.2141] [INSPIRE].

[35] F. Dominguez, C. Marquet, B.-W. Xiao and F. Yuan, Universality of unintegrated gluon distributions at small x, Phys. Rev. D 83 (2011) 105005 [arXiv:1101.0715] [INSPIRE].

[36] P. Kotko, K. Kutak, C. Marquet, E. Petreska, S. Sapeta and A. van Hameren, Improved TMD factorization for forward dijet production in dilute-dense hadronic collisions, JHEP 09 (2015) 106 [arXiv:1503.03421] [INSPIRE].

[37] C. Marquet, E. Petreska and C. Roiesnel, Transverse-momentum-dependent gluon distributions from JIMWLK evolution, JHEP 10 (2016) 065 [arXiv: 1608. 02577] [INSPIRE].

[38] A. van Hameren, P. Kotko, K. Kutak, C. Marquet, E. Petreska and S. Sapeta, Forward di-jet production in $p+P b$ collisions in the small-x improved TMD factorization framework, JHEP 12 (2016) 034 [arXiv: 1607.03121] [INSPIRE].

[39] A.H. Mueller, B.-W. Xiao and F. Yuan, Sudakov resummation in small-x saturation formalism, Phys. Rev. Lett. 110 (2013) 082301 [arXiv:1210.5792] [INSPIRE].

[40] A.H. Mueller, B.-W. Xiao and F. Yuan, Sudakov double logarithms resummation in hard processes in the small-x saturation formalism, Phys. Rev. D 88 (2013) 114010 [arXiv: 1308.2993] [INSPIRE].

[41] J. Zhou, The evolution of the small-x gluon TMD, JHEP 06 (2016) 151 [arXiv:1603.07426] [INSPIRE].

[42] I. Balitsky and A. Tarasov, Rapidity evolution of gluon TMD from low to moderate $x$, JHEP 10 (2015) 017 [arXiv: 1505.02151] [INSPIRE].

[43] A. Schäfer and J. Zhou, A note on the scale evolution of tri-gluon correlations, arXiv: 1308.4961 [INSPIRE].

[44] J. Zhou, Transverse single spin asymmetries at small $x$ and the anomalous magnetic moment, Phys. Rev. D 89 (2014) 074050 [arXiv: 1308.5912] [INSPIRE].

[45] D. Boer, M.G. Echevarria, P. Mulders and J. Zhou, Single spin asymmetries from a single Wilson loop, Phys. Rev. Lett. 116 (2016) 122001 [arXiv:1511.03485] [INSPIRE].

[46] L. Szymanowski and J. Zhou, The spin dependent odderon in the diquark model, Phys. Lett. B 760 (2016) 249 [arXiv: 1604.03207] [INSPIRE]. 
[47] Y. Hatta, B.-W. Xiao and F. Yuan, Probing the small-x gluon tomography in correlated hard diffractive dijet production in deep inelastic scattering, Phys. Rev. Lett. 116 (2016) 202301 [arXiv: 1601.01585] [INSPIRE].

[48] Y. Hagiwara, Y. Hatta and T. Ueda, Wigner, Husimi and generalized transverse momentum dependent distributions in the color glass condensate, Phys. Rev. D 94 (2016) 094036 [arXiv: 1609.05773] [INSPIRE].

[49] J. Zhou, Elliptic gluon generalized transverse-momentum-dependent distribution inside a large nucleus, Phys. Rev. D 94 (2016) 114017 [arXiv:1611.02397] [InSPIRE].

[50] E. Iancu and A.H. Rezaeian, Elliptic flow from color-dipole orientation in $p p$ and $p A$ collisions, Phys. Rev. D 95 (2017) 094003 [arXiv: 1702.03943] [INSPIRE].

[51] D. Boer, S. Cotogno, T. van Daal, P.J. Mulders, A. Signori and Y.-J. Zhou, Gluon and Wilson loop TMDs for hadrons of spin $\leq 1$, JHEP 10 (2016) 013 [arXiv:1607.01654] [INSPIRE].

[52] F. Gelis and J. Jalilian-Marian, Photon production in high-energy proton nucleus collisions, Phys. Rev. D 66 (2002) 014021 [hep-ph/0205037] [inSPIRE].

[53] T.C. Rogers and P.J. Mulders, No generalized TMD-factorization in hadro-production of high transverse momentum hadrons, Phys. Rev. D 81 (2010) 094006 [arXiv:1001.2977] [INSPIRE].

[54] A. Schäfer and J. Zhou, Transverse single spin asymmetry in direct photon production in polarized pA collisions, Phys. Rev. D 90 (2014) 034016 [arXiv:1404.5809] [InSPIRE].

[55] A. Schäfer and J. Zhou, Color entanglement for $\gamma$-jet production in polarized $p$ A collisions, Phys. Rev. D 90 (2014) 094012 [arXiv: 1406.3198] [INSPIRE].

[56] J. Zhou, Transverse single spin asymmetry in Drell-Yan production in polarized $p A$ collisions, Phys. Rev. D 92 (2015) 014034 [arXiv: 1502.02457] [InSPIRE].

[57] A.H. Mueller, Parton saturation at small $x$ and in large nuclei, Nucl. Phys. B 558 (1999) 285 [hep-ph/9904404] [INSPIRE].

[58] D. Boer, TMD evolution of the Sivers asymmetry, Nucl. Phys. B 874 (2013) 217 [arXiv: 1304.5387] [INSPIRE].

[59] S.M. Aybat and T.C. Rogers, TMD parton distribution and fragmentation functions with QCD evolution, Phys. Rev. D 83 (2011) 114042 [arXiv:1101.5057] [InSPIRE].

[60] D. Boer, Linearly polarized gluon effects in unpolarized collisions, PoS (QCDEV2015) 023 [arXiv: 1510.05915] [INSPIRE].

[61] J. Collins, L. Gamberg, A. Prokudin, T.C. Rogers, N. Sato and B. Wang, Relating transverse momentum dependent and collinear factorization theorems in a generalized formalism, Phys. Rev. D 94 (2016) 034014 [arXiv: 1605.00671] [INSPIRE].

[62] K.J. Golec-Biernat and M. Wüsthoff, Saturation effects in deep inelastic scattering at low $Q^{2}$ and its implications on diffraction, Phys. Rev. D 59 (1998) 014017 [hep-ph/9807513] [INSPIRE].

[63] G. Parisi and R. Petronzio, Small transverse momentum distributions in hard processes, Nucl. Phys. B 154 (1979) 427 [inSPIRE].

[64] G. Bozzi, S. Catani, D. de Florian and M. Grazzini, The $q_{T}$ spectrum of the Higgs boson at the LHC in QCD perturbation theory, Phys. Lett. B 564 (2003) 65 [hep-ph/0302104] [INSPIRE]. 